\title{
Modeling Insulin-Glucose Dynamics During Insulin Induced Hypoglycemia. Evaluation of Glucose Counterregulation
}

\author{
BORIS P. KOVATCHEV ${ }^{a} *$, LEON S. FARHY ${ }^{\mathrm{b}}$, DANIEL J. COX ${ }^{\mathrm{a}}$, MARTIN STRAUME ${ }^{\mathrm{c}}$, \\ VLADIMIR I. YANKOV ${ }^{a}$, LINDA A. GONDER-FREDERICK ${ }^{a}$ and WILLIAM L. CLARKE ${ }^{a}$ \\ "University of Virginia Health Sciences Center; ${ }^{b}$ Sofia University "St. Kliment Ohridski", Faculty of Mathematics; "NSF Center for \\ Biological Timing, University of Virginia Health Sciences Center
}

(Received 27 July 1998; In final form 21 October 1998)

\begin{abstract}
A dynamical network model of insulin-glucose interactions in subjects with Type I Diabetes was developed and applied to data sets for 40 subjects. Each data set contained the amount of dextrose + insulin infused and blood glucose (BG) determinations, sampled every 5 minutes during a one-hour standardized euglycemic hyperinsulinemic clamp and a subsequent one-hour BG reduction to moderate hypoglycemic levels. The model approximated the temporal pattern of $\mathrm{BG}$ and on that basis predicted the counterregulatory response of each subject. The nonlinear fits explained more than $95 \%$ of the variance of subjects' BG fluctuations, with a median coefficient of determination $97.7 \%$. For all subjects the model-predicted counterregulatory responses correlated with measured plasma epinephrine concentrations. The observed nadirs of BG during the tests correlated negatively with the model-predicted insulin utilization coefficient $(r=-0.51, p<0.001)$ and counterregulation rates $(r=-0.63, p<0.001)$. Subjects with a history of multiple severe hypoglycemic episodes demonstrated slower onset of counterregulation compared to subjects with no such history $(p<0.03)$.
\end{abstract}

Keywords: Dynamic models, hypoglycemia, counterregulation, hyperinsulinemic clamp

Insulin Dependent Diabetes Mellitus (IDDM) occurs when the pancreas produces insufficient insulin to prevent hyperglycemia, necessitating administration of exogenous insulin by injection. Excessive insulin, relative to metabolic needs, leads to low blood glucose (BG) or hypoglycemia, $B G<3.9 \mathrm{mM}$ as defined by the DCCT Study Group (1993). In most individuals with IDDM, low BG triggers the release of counterregulatory hormones. This in turn prompts the release of stored glucose into the bloodstream to restore euglycemia. However, in IDDM subjects, the ability to counterregulate is frequently impaired by factors such as long-standing diabetes, autonomic neuropathy, and intensive therapy (Amiel et al., 1987, 1988; Cryer et al., 1985). Insufficient or absent counterregulatory responses allow $\mathrm{BG}$ to

${ }^{*}$ Corresponding Author: University of Virginia Health Sciences Center, Box 137, Charlottesville, VA 22908, USA: Tel: (804)-924-8656; Fax: (804)-924-8652; E-mail: bpk2u@ Virginia.edu 
fall further until stupor, unconsciousness or seizure occurs. This condition, referred to as severe hypoglycemia $(\mathrm{SH})$, is responsible for four percent of the deaths among patients with IDDM (DCCT Study Group, 1991). The risk for SH in IDDM has been attributed not only to relative insulin excess, but also to impaired glucose counterregulation (Cryer et al., 1985; Gerich, 1988; White et al., 1983).

Glucose metabolism has been studicd with isotopic tracer methods in animal and human studies (Brier et al., 1977; Hetenyi, Ninomiya and Wrenshall, 1966) and described by several mathematical models that included network modeling of glucose metabolism in normal ideal man (Guyton et al., 1978) and diabetic dogs (Yamasaki, Tiran and Albisser, 1984), and multicompartment models (Insel et al., 1975; Steele, Rostami and Altszuler, 1974). The minimal model, suggested as an alternative of hyperinsulinemic euglycemic clamping for measuring insulin sensitivity in vivo (Bergman et al., 1979), received considerable attention, support and critiques (Bergman et al., 1987; Cobelli et al., 1986; Cobelli, Brier and Ferrannini, 1990; Mari, 1997; Quon et al., 1994). While some of these models focused specifically upon the role of the liver (Carson and Cramp, 1976), investigation of counterregulation through insulin infusion has typically been pursued in two ways: 1) during the induction of hypoglycemia various hormones are sampled to determine whether their levels increase as BG falls, 2) whether or not BG spontaneously rises or platcaus despite the continual infusion of regular insulin (Bolli et al., 1984). These basic approaches to describing glucose counterregulation included quantifying plasma hormonal concentrations, but they did not yield a precise mathematical model of glucose counterregulation.

In this manuscript we present a mathematical model of insulin-glucose dynamics during a euglycemic hyperinsulinemic clamp and subsequent reduction of $B G$ to hypoglycemic levels. Using this model, we approximated the temporal pattern of each subject's BG fluctuations and evaluated parameters of insulin and glucose sensitivity. On that basis we computed dynamic estimates for the onset and rate of these subject's counterregulatory responses. These results were verified by correlating for each subject the model-estimated counterregulatory dynamics with subsequently analyzed plasma epinephrine concentrations and applied to the study of relationships and group effects pertinent to $\mathrm{SH}$.

\section{RESEARCH DESIGN AND METHODS}

\section{Subjects}

Forty subjects were recruited through newsletters, notices posted in diabetes clinics and direct physician referral. All subjects had to have diabetes for at least two ycars and have taken insulin since the time of diagnosis. There were 16 males and 24 females, with mean age $35.5 \mathrm{yr}(\mathrm{SEM}=1.3)$, mean duration of disease $16.9 \mathrm{yr}(\mathrm{SEM}=1.5)$, mean insulin units/kilogram per day $0.59(\mathrm{SEM}=0.03)$, and mean glycosylated hemoglobin $8.6 \%$ (SEM $=$ $0.3)$. The non-diabetic range for the glycosylated hemoglobin assay was 4.4 to $6.9 \%$. Twenty-five subjects reported a history of multiple $\mathrm{SH}$ episodes in the past year while 15 subjects had no such history.

\section{Procedure}

All subjects attended orientation meetings and signed consent forms. To ensure that subjects' BGs were not in a low range $(<3.9 \mathrm{mM})$ for $72 \mathrm{hrs}$ prior to the study, their insulin dose was reduced by $10 \%$ and long acting insulin was discontinued $36 \mathrm{hrs}$ prior to the study. Subjects were instructed to eat prophylactically $10 \mathrm{~g}$ of glucose whenever $\mathrm{BG}$ was $\leq 5.6 \mathrm{mM}$ and were required to test their $B G$ five times a day ( $1 \mathrm{hr}$ before each meal, at bedtime and 4 hrs into their sleep). If low BG occurred, the study was rescheduled. Subjects were admitted to the University of Virginia General Clinical Research Center. Upon admission, subjects were given a physical exam, including an assessment for autonomic neuropathy. $\mathrm{BG}$ was maintained overnight between 5.6-8.3 $\mathrm{mM}$ with intravenous regular human insulin as per a 


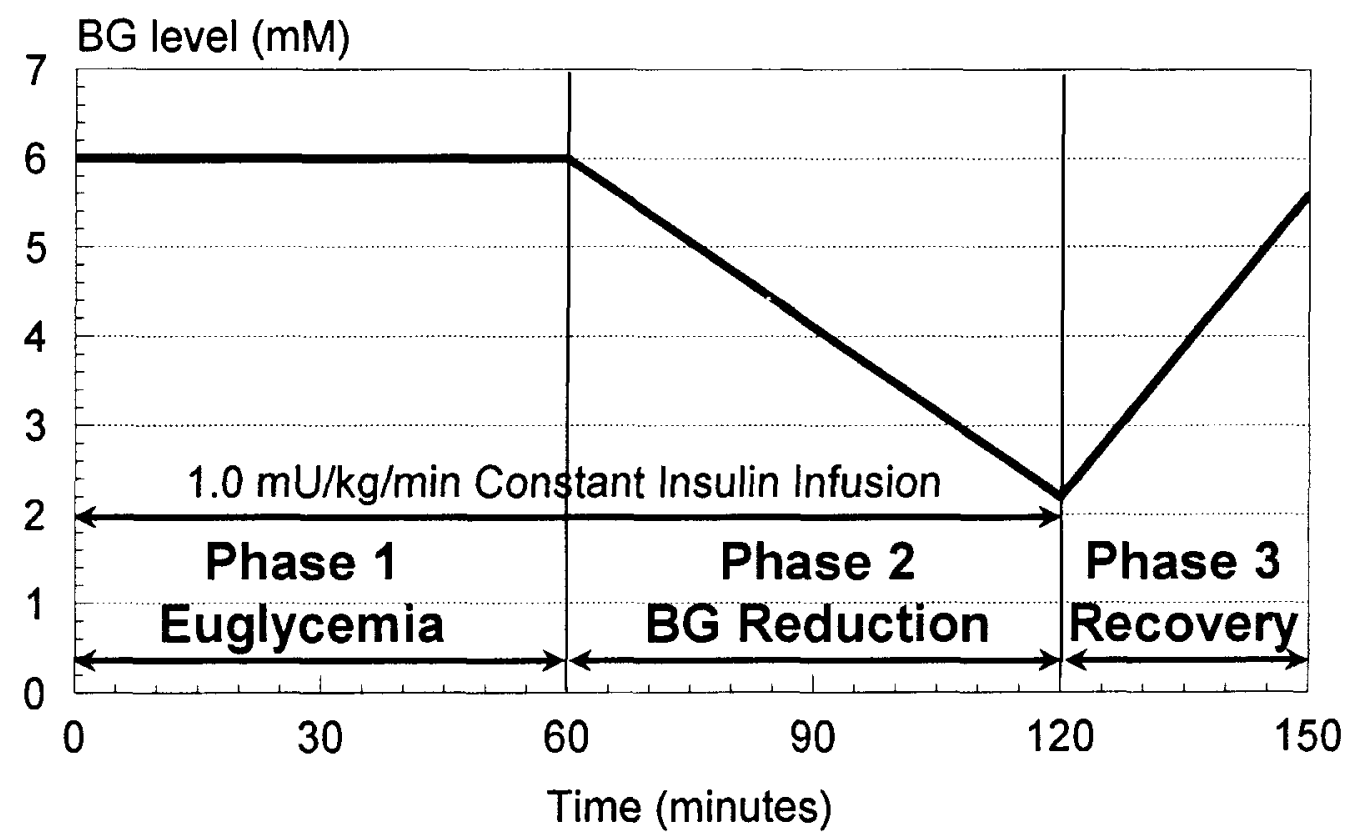

FIGURE 1 Design of the study: During Phase $1 \mathrm{BG}$ is maintained between 5.6 and $8.3 \mathrm{mM}$. During Phase $2 \mathrm{BG}$ is steadily lowered to a target level of $2.2 \mathrm{mM}$ by varying the dextrose infusion.

previously published insulin infusion protocol (Bolli et al., 1984). Subjects were given dinner and a bedtime snack the evening before the study, but remained fasting on the morning of the study. No caffeinated beverages were consumed after hospital admission.

On the morning of the study, IV's were placed in the nondominant forearm. Insulin was continuously infused at a constant rate of $1.0 \mathrm{mU} / \mathrm{kg} / \mathrm{min}$ and a $20 \%$ dextrose solution was infused at a variable rate to maintain $\mathrm{BG}$ at $6 \mathrm{mM}$. Figure 1 presents the design of the study.

During Phase 1 (euglycemia) BG was maintained between 5.6 and $8.3 \mathrm{mM}$. During Phase 2 (BG reduction), $\mathrm{BG}$ was steadily lowered to a target level of $2.2 \mathrm{mM}$ by varying the dextrose infusion. Adjustments in dextrose infusion were made every $5 \mathrm{~min}$. The insulin infusion was discontinued during Phase 3 (recovery). The protocol was discontinued if manifestations of SH occurred (e.g. severe lethargy, disorientation, confusion or inappropriate behavior). Arterialized blood (achieved by warming the hand in a heated glove to $50^{\circ} \mathrm{C}$ ) was sampled for glucose concentration every $5 \mathrm{~min}$ and for plasma epinephrine concentration every $10 \mathrm{~min}$.

\section{Mathematical Model of Insulin-Glucose Dynamics}

A dynamical network model was developed to describe $B G$ dynamics as a function of two principal temporal variables, insulin and dextrose infusion. Since the subjects did not eat and their physical activity was negligible during the study, neither of these parameters was assumed to influence BG. In addition to the two principal variables, a counterregulatory response was anticipated at lower BG levels. The network of functional interactions is shown in Figure 2.

The system was defined in terms of three timedependent state variables: 1) BG level, 2) insulin infusion, and 3) dextrose infusion. A network of processes provides the functional regulatory interactions responsible for $\mathrm{BG}$ control: $\mathrm{BG}$ level was positively affected by dextrose infusion (process $\mathbf{D}$ ), as well as by the potential for replenishment when $B G$ is low from available liver stores (process $\boldsymbol{C R}$ ). The negative effect on BG level by insulin injections is denoted by process I. A regulatory loop (Reg) between liver stores and BG is implemented by way 


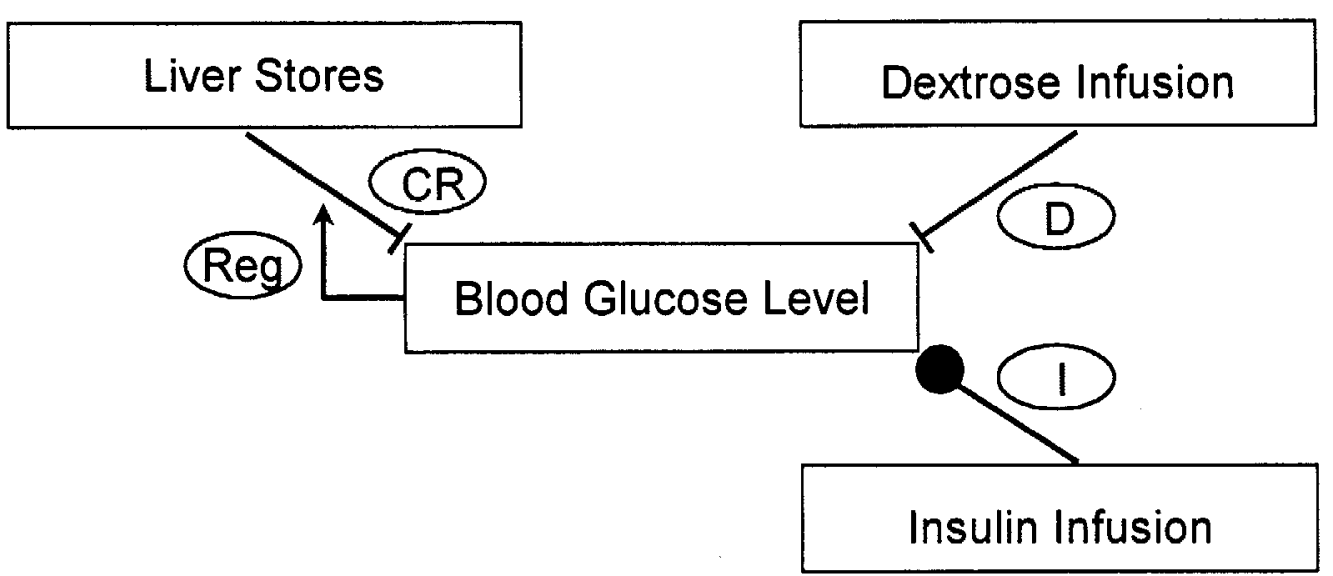

FIGURE 2 Network of functional interactions during hyperinsulinemic clamp: BG levels is positively affected by the dextrose infusion (D) and by the potential for replenishment when BG is low $(\boldsymbol{C R})$, and negatively affected by the insulin infusion (I). A regulatory loop (Reg) between liver stores and BG inhibits the process $\boldsymbol{C} R$ at elevated BG levels.

of inhibition of process $\boldsymbol{C R}$ by elevated BG levels (i.e., a release from inhibition of process $\boldsymbol{C R}$ below some threshold BG level, thus providing for counterregulatory recovery from low BG levels by recruitment of available liver stores). Time rates of change of $\mathrm{BG}$ (i.e., $\mathrm{d}[\mathrm{BG}] / \mathrm{d} t$ ) were described by a nonlinear ordinary differential equation. The counterregulatory response was modeled as a release of glucose from a multi-compartment storage pool. It was assumed that during the first phase no counterregulatory response occurred. This permitted estimation of each individual's insulin-glucose dynamics parameters. During the second phase, an adaptive stepwise procedure was used to determine the onset and the rate of counterregulation.

\section{Phase 1: Maintained euglycemia}

It was assumed that: 1) the dextrose infusion influenced BG positively through an unknown dextrose conversion parameter $\boldsymbol{a}$, and 2) BG decay rate was inversely proportional to the $\mathrm{BG}$ level, through an unknown insulin utilization parameter $\boldsymbol{b}$. This led to the following nonlinear differential equation for the BG time rate of change:

$$
\frac{\mathrm{dBG}(t)}{\mathrm{d} t}=a \cdot D(t)-b \cdot \frac{\mathrm{BG}(t)}{\varepsilon+\mathrm{BG}(t)^{2}} . I
$$

where $D(t)$ is the variable dextrose infusion rate ( $\mathrm{mg} / \mathrm{kg} / \mathrm{min}$ ) and $I$ is the constant insulin infusion. The inverse proportion $\mathrm{BG}(t) /\left(\varepsilon+\mathrm{BG}(t)^{2}\right)$, where $\varepsilon$ is a small constant, was used instead of standard $1 / \mathrm{BG}(t)$ for a better computational stability.

\section{Phase 2: BG reduction}

During the second phase of the test counterregulation was anticipated and equation [A] was expanded by an additional term:

$$
\frac{\mathrm{dBG}(t)}{\mathrm{d} t}=a \cdot D(t)-b \cdot \frac{\mathrm{BG}(t)}{\varepsilon+\mathrm{BG}(t)^{2}} \cdot I+C R(t)
$$

We allowed the counterregulation term $C R(t)$ to be a uni- or bi-modal function, corresponding to one- or two-compartment modeling. We would not impose a specific analytical form on the counterregulation function. In general it needs to be a positive pulsatile function that, depending on the subject's data, has one, two (or possibly more) additive components: $C R(t)=C R_{1}(t)$, or $C R(t)=$ $C R_{1}(t)+C R_{2}(t)$. For this particular application in order to be able to approximate our data, we suggest each counterregulation component to be defined by:

$$
C R_{i}(t)=\left\{\begin{array}{rr}
0 & \text { when } t<T_{i} \\
C_{i} r_{i}^{2} \cdot\left(t-T_{i}\right) \cdot \exp \left(-r_{i} \cdot\left(t-T_{i}\right)\right) & \text { when } t \geq T_{i}, i=1,2 .
\end{array}\right.
$$


This way the function $C R_{1}(t)$, would be zero when $t<T_{1}$ and would increase at time $T_{1}$. Thus, the parameter $T_{1}$ would interpreted as time of onset of counterregulation, while the product $C_{1}, r_{1}^{2}$ would be the counterregulation slope at onset (the derivative at time $\left.t=T_{1}\right)$. The same would be valid for the second component $C R_{2}(t)$. The analytical form of $C R(t)$ was carefully selected to allow for this physiological interpretation of its parameters, however it is not restricted and other functions that meet certain mathematical requirements would be suitable descriptors of counterregulatory responses.

\section{Parameter Estimation}

The input data for the model were each subject's dextrose infusion records and corresponding BG levels, each of which were recorded every 5 minutes. An automated algorithm for analysis of these data was developed as follows:

Prior to identification by the algorithm of onset of counterregulation, each subject's parameters $\boldsymbol{a}$ and $\boldsymbol{b}$ were estimated, along with a maximumlikelihood estimate of their initial BG level. This was accomplished by a modified Gauss-Newton nonlinear least squares parameter estimation algorithm (Johnson and Frasier, 1985; Straume, FrasierCadoret and Johnson, 1991) in which the differential equation [A] was integrated numerically for $\mathrm{BG}(t)$ by a fourth-order Runge-Kutta method (Press et al., 1989). Applied to each subjects' data set, this procedure successfully evaluated individually for each subject these characteristics of the dynamics of dextrose utilization and $\mathrm{BG}$ elimination during euglycemia and descent into hypoglycemia prior to onset of counterregulation.

The algorithm was initialized to consider first only those time points in which there was clearly no potential for counterregulation i.e., (those points comprising the euglycemic phase of the experiment; Phase 1 of Figure 1). The parameters $\boldsymbol{a}$ and $\boldsymbol{b}$ and the initial BG level were nonlinear least squares estimated to this subset of data, followed by evaluation of the standard deviation of fit to the $\mathrm{BG}$ data. At this point, the difference between the observed
BG level for the next time point and the BG level predicted by the model in the absence of counterregulation was evaluated. If this difference was less than two standard deviations of fit, this next observed BG level was considered to be prior to onset of counterregulation and was included as an additional point for estimation of the parameters $\boldsymbol{a}$ and $\boldsymbol{b}$ and an initial BG level by equation [A]. This process was repeated iteratively until a $B G$ level was identified that differed from the predicted value by more than two standard deviations of fit, indicating onset of counterregulation. From this point onward, the parameters $a$ and $b$ and the estimated initial BG level were fixed, onset of counterregulation (parameter $T_{1}$ ) was defined as the time of the previous time point, and the model began fitting to differential equation [B]. The process again proceeded iteratively one point at a time until either the remainder of the data set was successfully considered or until the need for a second component of the counterregulatory response was identified (in the same manner as above).

\section{RESULTS}

The average $B G$ level during the first euglycemic phase (Phase 1) of the study was $6.3 \mathrm{mM}$ $(\mathrm{SEM}=0.1$ ). The average nadir of $\mathrm{BG}$ reached during the second phase of the study was $2.5 \mathrm{mM}$ $(\mathrm{SEM}=0.08)$. The average plasma epinephrine concentration during Phase 1 was 52 (SEM =6.3). During Phase 2 the average epinephrine peak was 367 $(\mathrm{SEM}=44)$.

\section{Goodness-of-fit of the Model}

The algorithm was applied to the data sets of each of the 40 subjects. The accuracy of the data fit was tested by the coefficient of determination, usually interpreted as the percentage of the total variation of the dependent variable around its mean that is explained by the fitted model (Kvalseth, 1985), and by the mean square error (MSE) per data point. Since the model is nonlinear and a standard ANOVA $p$-value cannot be computed, the goodness-of-fit 
of each model was evaluated by the closeness of its coefficient of determination to $100 \%$ (note that our model is intrinsically nonlinear, and therefore the usual F-statistic and its significance level cannot be used). The median coefficient of determination across subjects was $97.7 \%$, with a maximum of $99.7 \%$ and a minimum of $86.5 \%$. The median MSE of the model fits was $0.16 \mathrm{mM}$, with a range from 0.07 to $0.44 \mathrm{mM}$, i.e. for all subjects the model-predicted BG fluctuations were within $0.44 \mathrm{mM}$ from the observed $\mathrm{BG}$ values, with half of the subjects within $0.16 \mathrm{mM}$. This indicates an extremely good model fit for all subjects. Table I presents goodness-of-fit data for all subjects ordered by their coefficients of determination. Seven subjects had coefficients of determination above $99 \%$, while only 2 subjects had coefficients of determination below $90 \%$.

In order to better illustrate our model Figure 3 presents a sample (not the best but above average) data fit for subject $\# 9$ whose coefficient of determination was $98.8 \%, \mathrm{MSE}=0.14 \mathrm{mM}$.

TABLE I Model goodness-of-fit: Coefficients of determination (CD) and mean square errors (MSE) for all participants in the study

\begin{tabular}{lcclllllllll}
\hline$\#$ & CD & MSE & $\#$ & CD & MSE & $\#$ & CD & MSE & $\#$ & CD & MSE \\
\hline 1 & $99.7 \%$ & 0.07 & 11 & $98.6 \%$ & 0.19 & 21 & $97.6 \%$ & 0.20 & 31 & $95.1 \%$ & 0.15 \\
$2 \mathrm{sh}$ & $99.7 \%$ & 0.07 & 12 & $98.6 \%$ & 0.17 & $22 \mathrm{sh}$ & $97.6 \%$ & 0.17 & $32 \mathrm{sh}$ & $94.9 \%$ & 0.23 \\
$3 \mathrm{sh}$ & $99.5 \%$ & 0.07 & 13 & $98.5 \%$ & 0.18 & 23 & $97.4 \%$ & 0.19 & $33 \mathrm{sh}$ & $94.7 \%$ & 0.22 \\
$4 \mathrm{sh}$ & $99.4 \%$ & 0.10 & $14 \mathrm{sh}$ & $98.5 \%$ & 0.21 & 24 & $97.4 \%$ & 0.16 & $34 \mathrm{sh}$ & $94.4 \%$ & 0.25 \\
$5 \mathrm{sh}$ & $99.4 \%$ & 0.08 & $15 \mathrm{sh}$ & $98.3 \%$ & 0.15 & $25 \mathrm{sh}$ & $97.2 \%$ & 0.16 & $35 \mathrm{sh}$ & $93.0 \%$ & 0.32 \\
$6 \mathrm{sh}$ & $99.3 \%$ & 0.11 & $16 \mathrm{sh}$ & $98.3 \%$ & 0.16 & $26 \mathrm{sh}$ & $96.9 \%$ & 0.22 & $36 \mathrm{sh}$ & $92.1 \%$ & 0.39 \\
7 & $99.2 \%$ & 0.07 & 17 & $98.1 \%$ & 0.13 & 27 & $96.9 \%$ & 0.16 & $37 \mathrm{sh}$ & $90.9 \%$ & 0.15 \\
$8 \mathrm{sh}$ & $98.9 \%$ & 0.16 & 18 & $97.8 \%$ & 0.16 & $28 \mathrm{sh}$ & $96.5 \%$ & 0.13 & $38 \mathrm{sh}$ & $90.9 \%$ & 0.39 \\
$9^{*}$ & $98.8 \%$ & 0.14 & $19 \mathrm{sh}$ & $97.8 \%$ & 0.13 & 29 & $95.6 \%$ & 0.22 & $39 \mathrm{sh}$ & $88.5 \%$ & 0.44 \\
$10 \mathrm{sh}$ & $98.8 \%$ & 0.12 & $20 \mathrm{sh}$ & $97.7 \%$ & 0.17 & $30 \mathrm{sh}$ & $95.6 \%$ & 0.20 & 40 & $86.5 \%$ & 0.19 \\
\hline
\end{tabular}

*Dat:a used for Figure 3 .

"sh" next to a subject's number marks the subjects who reported problems with severe hypoglycemia in the past year.

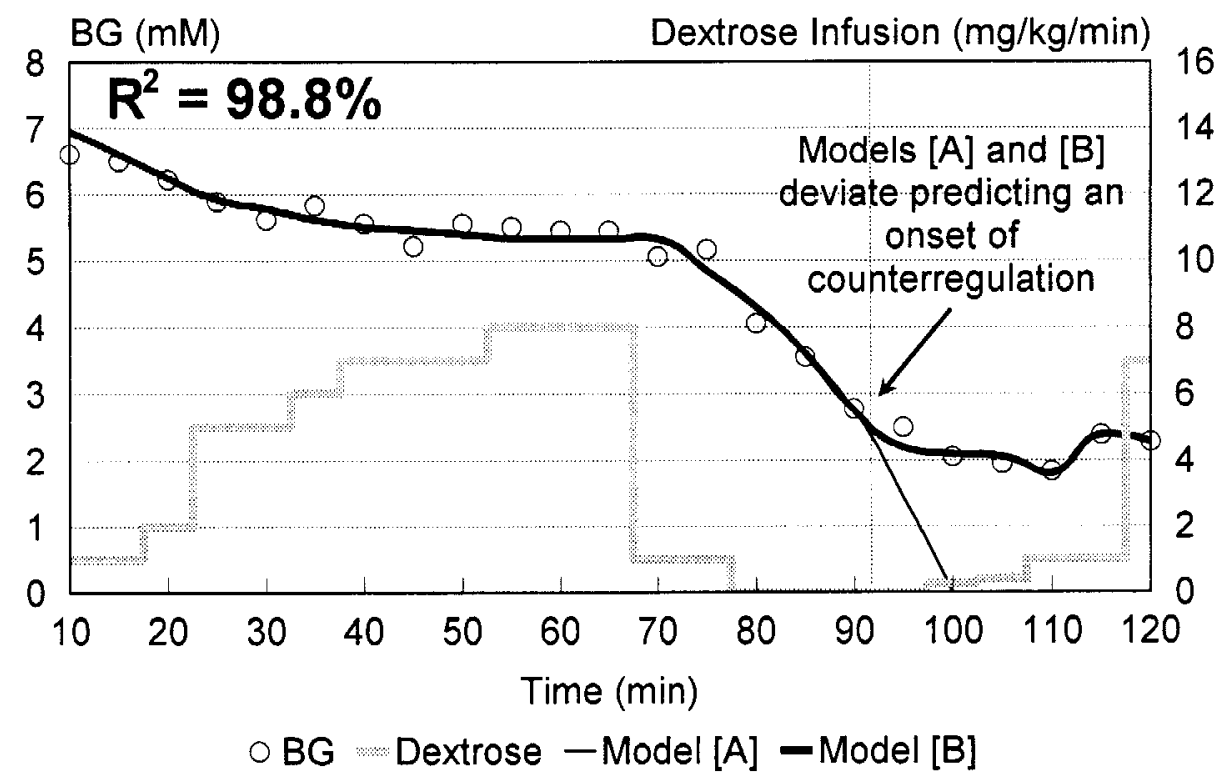

FIGURL 3 Model fit: The left y-axis (BG (mM)) refers to three variables: 1) The BG data ploted by circles. 2) The full model fit. based on equation $[B]$ (thick black linc). 3) The model-predicted BG decay if counterregulation did not occur, based on equalion $[A]$ (thin black line). The dextrose infusion is ploted along the right y-axis as a stepwise grey line. 
The $x$-axis of Figure 3 is the elapsed time in minutes. The left $y$-axis $(B G(m M))$ refers to three variables: 1) The BG data plotted by circles. 2) The full model fit, based on equation [B] (thick black line). 3) The model-predicted BG decay if counterregulation did not occur, based on equation [A] (thin black line). The dextrose infusion that is plotted along the right $\mathrm{y}$-axis, is represented by a stepwise grey line, is a constant within each 5-minute interval and is adjusted every 5 minutes. The figure includes this subject's coefficient of determination $R^{2}=98.8 \%$ and MSE $=0.14 \mathrm{mM}$.

\section{Counterregulation}

The rate of counterregulation of each subject was estimated in units equivalent to $\mathrm{mg} / \mathrm{kg} / \mathrm{min}$ dextrose infusion, on the basis of the difference between models [B] and [A], as explained above (Parameter Estimation). In Figure 3 the counterregulation would be equivalent to dextrose infusion needed to elevate the $B G$ level fit from the thin to the thick black line, i.e. from model [A] to model [B]. Consequently the onset of counterregulation will be the point where these two lines split, i.e. shortly after minute 90 in Figure 3.

An external validation of the predicted counterregulation rate was done using its correlations with the corresponding epinephrine data for each subject. Table II presents the correlation coefficients between model-predicted counterregulation and logarithm of epinephrine concentration together with their significance level. For subjects who counterregulated the median correlation coefficient was 0.82 , range from 0.47 to 0.97 with all correlations significant at $p=0.05$. The model estimated that four subjects did not counterregulate and this was confirmed by their non-increasing plasma epinephrine concentrations (\#17, \#36, \#37 and \#39 in Table II).

Figure 4 illustrates the relationship between the model-predicted counterregulation and the plasma epinephrine concentrations, recorded every $10 \mathrm{~min}$ utes, for subject \#9 whose data and model curves were plotted in Figure 3. As with Figure 3, the $x^{-}$ axis represents the elapsed time in minutes. The left $y$-axis refers to logarithm of epinephrine concentration that is measured every 10 minutes and presented by a grey stepwise line. The right y-axis represents the model-evaluated counterregulation in units equivalent to $\mathrm{mg} / \mathrm{kg} / \mathrm{min}$ dextrose infusion. The counterregulatory response of this subject began shortly after minute 90 and then increased rapidly with a small setback at minute 105 . The correlation between epinephrine and counterregulation was significant, $R=0.91, p<0.001$.

\section{Parameters of the Model}

As we discussed in the previous section, our model has four essential parameters. Two of them, glucose

TABLE II Correlations $(R)$ between counterregulation rates and logarithm of epinephrine concentrations together with their significance levels ( $p)$

\begin{tabular}{|c|c|c|c|c|c|c|c|c|c|c|c|}
\hline$\#$ & $R$ & $p$ & $\#$ & $R$ & $p$ & $\#$ & $R$ & $p$ & $\#$ & $R$ & $p$ \\
\hline 1 & 0.89 & 0.000 & 11 & 0.88 & 0.000 & 21 & 0.93 & 0.000 & 31 & 0.81 & 0.000 \\
\hline $2 \mathrm{sh}$ & 0.55 & 0.016 & 12 & 0.85 & 0.000 & $22 \mathrm{sh}$ & 0.72 & 0.002 & $32 \mathrm{sh}$ & 0.47 & 0.050 \\
\hline $3 \mathrm{sh}$ & 0.96 & 0.000 & 13 & 0.62 & 0.020 & 23 & 0.97 & 0.000 & $33 \mathrm{sh}$ & 0.88 & 0.000 \\
\hline $4 \mathrm{sh}$ & 0.96 & 0.000 & $14 \mathrm{sh}$ & 0.95 & 0.000 & 24 & 0.82 & 0.001 & $34 \mathrm{sh}$ & 0.88 & 0.000 \\
\hline $5 \mathrm{sh}$ & 0.71 & 0.003 & $15 \mathrm{sh}$ & 0.95 & 0.000 & $25 \mathrm{sh}$ & 0.62 & 0.004 & $35 \mathrm{sh}$ & 0.75 & $0.00 \mathrm{I}$ \\
\hline $6 \mathrm{sh}$ & 0.48 & 0.048 & $16 \mathrm{sh}$ & 0.90 & 0.000 & $26 \mathrm{sh}$ & 0.94 & 0.000 & $36 \mathrm{sh}$ & \multicolumn{2}{|c|}{ No $C R$} \\
\hline 7 & 0.82 & 0.000 & 17 & \multicolumn{2}{|c|}{ No $C R$} & 27 & 0.94 & 0.000 & $37 \mathrm{sh}$ & \multicolumn{2}{|c|}{ No $C R$} \\
\hline $8^{*} \mathrm{sh}$ & & & 18 & 0.95 & 0.000 & $28 \mathrm{sh}$ & 0.89 & 0.000 & $38 \mathrm{sh}$ & 0.51 & 0.039 \\
\hline $9^{* *}$ & 0.91 & 0.000 & $19 \mathrm{sh}$ & 0.72 & 0.005 & 29 & 0.94 & 0.000 & $39 \mathrm{sh}$ & \multicolumn{2}{|c|}{ No $C R$} \\
\hline $10 \mathrm{sh}$ & 0.80 & 0.001 & $20 \mathrm{sh}$ & 0.82 & 0.000 & $30 \mathrm{sh}$ & 0.89 & 0.000 & 40 & 0.56 & 0.018 \\
\hline
\end{tabular}

No epinephrine data were available for subject $\# 8$

${ }^{* *}$ Data used for Figure 4. 


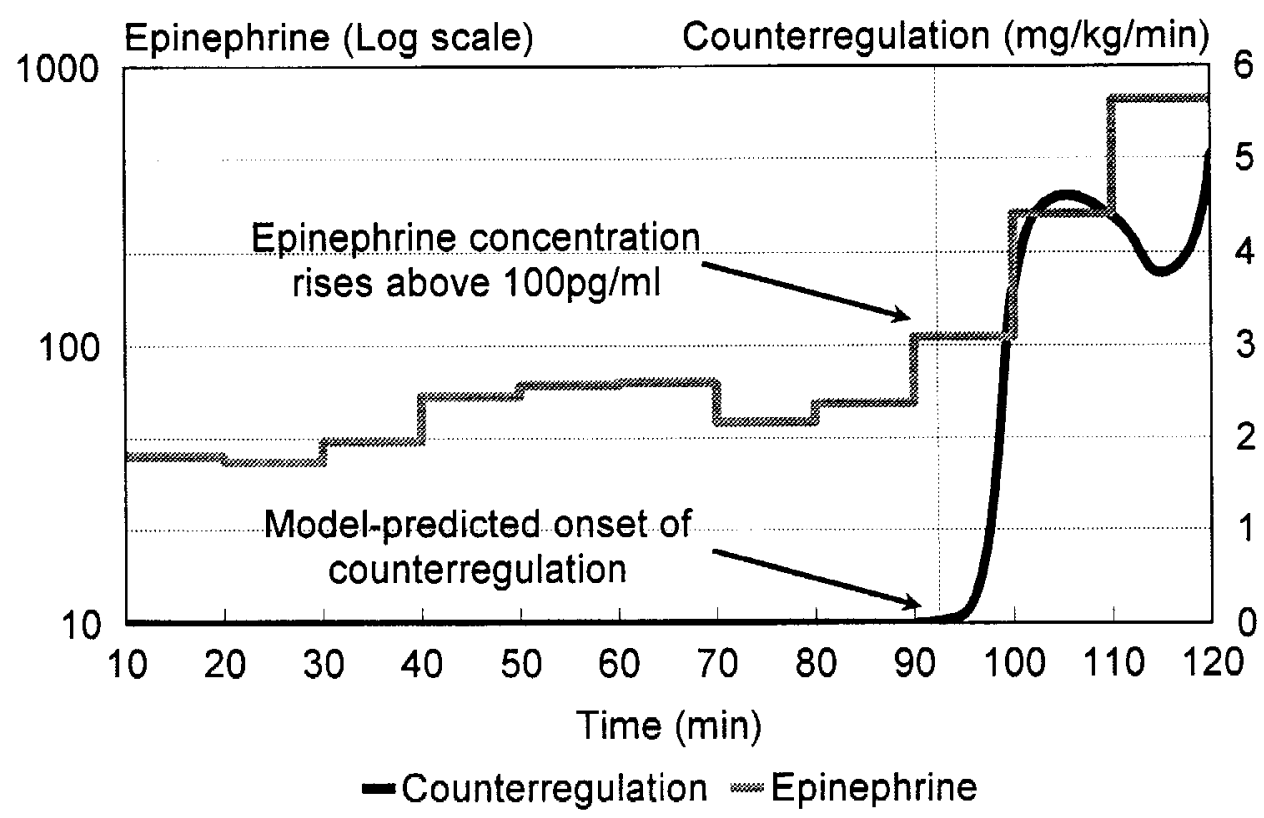

FIGURE 4 Model-predicted counterregulation and plasma epinephrine concentrations: The left y-axis refers to logarithm of epinephrine concentration that is measured every 10 minutes and presented by a grey stepwise line. The right $y$-axis represents the model-evaluated counterregulation in units equivalent to $\mathrm{mg} / \mathrm{kg} / \mathrm{min}$ dextrose infusion.

TABLE III Descriptive statistics for the model parameters

\begin{tabular}{lcc}
\hline Parameter & Mean & $\begin{array}{c}\text { Standard Error of } \\
\text { the Mcan }\end{array}$ \\
\hline Glucose conversion coefficient $a$ & 0.02 & 0.002 \\
Insulin utilization coefficient $b$ & 5.05 & 0.4 \\
Time of counterregulation onset $T_{1}(\mathrm{~min})$ & 114 & 1.9 \\
BG level at onset of counterregulation $(\mathrm{mM})$ & 3.85 & 0.15 \\
Average counterregulation rate $(\mathrm{mg} / \mathrm{kg} / \mathrm{min}$ dextrose) & 2.3 & 0.19 \\
Maximal counterregulation rate $(\mathrm{mg} / \mathrm{kg} / \mathrm{min}$ dextrose) & 3.8 & 0.33 \\
Countcregulation slope al onset $C_{1} \cdot r_{1}^{2}$ & 1.6 & 0.28 \\
\hline
\end{tabular}

conversion $a$ and insulin utilization coefficient $b$ were determined and fixed during the euglycemic Phase 1 of the study. The other two, time of counterregulation onset $T_{1}$ and the counterregulation slope at onset $C_{1} \cdot r_{1}^{2}$, were determined during Phase 2 . In addition, based on the model we computed the average and maximal counterregulation rate for each subject as well as his BG level at onset of counterregulation. Table III presents descriptive characteristics for these parameters.

Faster insulin utilization was associated with lower observed nadir of BG during the study as evidenced by the negative correlation of the insulin utilization coefficient with the nadir of $B G$, $R=-0.51(p<0.001)$. Lower nadir of $\mathrm{BG}$ was associated with higher counterregulation rate as evidenced by the negative correlations between nadir of $\mathrm{BG}$ and the average and maximum counterregulation rates, $R=-0.63(p<0.001)$ and $R=-0.64(p<$ $0.001)$ respectively. The average epinephrine response per subject correlated with the average counterregulation rate, $R=0.4, p=0.005$, while the maximum epinephrine response correlated with the maximal counterregulation, $R=0.4, p=0.006$. 
TABLE IVA Observed group effects

\begin{tabular}{lccc}
\hline Variable & $\begin{array}{c}\text { History of Multiple } \\
\text { SH, } N=25 \\
\text { Mean (SEM) }\end{array}$ & $\begin{array}{c}\text { No History of } \\
\text { SH, } N=15, \\
\text { Mean (SEM) }\end{array}$ & $p$ \\
\hline Age (years) & $37.0(1.7)$ & $33.0(1.8)$ & n.s. \\
Duration of diabetes (years) & $17.3(1.7)$ & $16.2(2.9)$ & n.s. \\
Insulin units/kilogram per day & $0.57(0.04)$ & $0.62(0.05)$ & n.s. \\
Glycosylated hemoglobin & $8.4(0.4)$ & $8.8(0.4)$ & n.s. \\
Nadir of BG during the test (mM) & $2.5(0.1)$ & $2.6(0.15)$ & n.s. \\
Average epinephrine concentration, Phase 2 & $130(28)$ & $176(25)$ & n.s. \\
Maximal epinephrine concentration, Phase 2 & $289(51)$ & $493(72)$ & 0.025 \\
\hline
\end{tabular}

TABLE IVB Model-estimated group effects

\begin{tabular}{|c|c|c|c|}
\hline Parameter & $\begin{array}{c}\text { History of Multiple } \\
\text { SH, } N=25 \\
\text { Mean (SEM) }\end{array}$ & $\begin{array}{l}\text { No History of } \\
\text { SH, } N=15 \\
\text { Mean (SEM) }\end{array}$ & $p$ \\
\hline Glucose conversion coefficient $a$ & $0.02(0.002)$ & $0.02(0.002)$ & n.s. \\
\hline Insulin utilization coefficient $b$ & $4.6(0.3)$ & $5.6(0.8)$ & n.s. \\
\hline Time of counterregulation onset $T_{1}$ (min) & $114(2.6)$ & $115(3.1)$ & n.s. \\
\hline $\begin{array}{l}\text { BG level at onset of counterregulation } \\
(\mathrm{mM})\end{array}$ & $3.8(0.18)$ & $3.9(0.3)$ & n.s. \\
\hline $\begin{array}{l}\text { Average counterregulation rate }(\mathrm{mg} / \mathrm{kg} / \mathrm{min} \\
\text { dextrose) }\end{array}$ & $2.1(0.22)$ & $2.6(0.39)$ & n.s. \\
\hline $\begin{array}{l}\text { Maximal counterregulation rate }(\mathrm{mg} / \mathrm{kg} / \mathrm{min} \\
\text { dextrose) }\end{array}$ & $3.4(0.36)$ & $4.5(0.67)$ & 0.13 \\
\hline Counterregulation slope at onset $C_{1}, r_{1}^{2}$ & $1.1(0.2)$ & $2.4(0.6)$ & 0.026 \\
\hline
\end{tabular}

\section{Group Effects}

The two groups of subjects, with and without a history of SH, did not differ in terms of age, duration of diabetes, insulin units/kilogram per day or glycosylated hemoglobin. During the test subjects from both groups reached similar nadirs of BG and had similar average epinephrine responses. Subjects with no history of SH demonstrated higher maximal epinephrine response, $493 \mathrm{pg} / \mathrm{ml}(\mathrm{SEM}=72)$ vs. $289 \mathrm{pg} / \mathrm{ml}(\mathrm{SEM}=51), p=0.025$. Table IVA summarizes these observations.

Based on the model, the two subject groups did not differ in terms of glucose conversion and insulin utilization coefficients, time and BG at onset of counterregulation, and average counterregulation rate. Subjects with no history of SH demonstrated greater counterregulation slope at onset, i.e. faster onset of counterregulation, $2.4(\mathrm{SEM}=0.6)$ vs. $1.1(\mathrm{SEM}=0.2), \quad p=0.026$ and marginally higher maximal counterregulation rate, $p=0.13$. Table IVB summarizes these results.

\section{DISCUSSION}

The deterministic differential equation model developed in the present study accounts, in a highly reliable manner, for the dynamics of both exogenous dextrose-infusion-rate-dependent changes in blood glucose levels as well as endogenous physiological counterregulation during euglycemic hyperinsulinemic clamping of patients with IDDM. During euglycemia in the absence of counterregulatory response, the model is mechanistically parameterized, individually and separately for each patient considered, in terms of two physiological processes: 1) the dextrose-to-blood glucose conversion efficiency and 2) the insulin utilization efficiency for elimination of blood glucose. The model was 
implemented during analysis to use an objective criterion for identifying the time of onset of physiological counterregulation at low blood glucose levels. Counterregulation was then parameterized, again, individually and separately for each patient considered, in terms of the rate and volume of counterregulatory response, as well as for the potential for bimodal counterregulation.

The objective analysis performed by this implementation of the model was successful in all 40 of the blood glucose-dextrose infusion data sets of IDDM patients considered in this study, typically accounting for greater than $95 \%$ of the observed variance in blood glucose time series. Additionally, the counterregulatory responses predicted by the model are consistent with observed plasma epinephrine concentrations, as indicated by the high correlation between the two. Interestingly, the modeling results suggest the previously unrecognized possibility that blood glucose counterregulation may be a multicomponent process (as a bimodal counterregulatory response was predicted for 22 of the 40 individuals examined).

Lower nadir of BG during the study was associated by our model with a faster insulin utilization. On the other hand, a lower nadir of $\mathrm{BG}$ prompted higher and more aggressive counterregulatory response, but did not result in clearly larger epinephrine response as evidenced by a non-significant nadir BG-epinephrine correlation $R=-0.2, p=0.1$.

Finally, our data indicated that a history of SH was associated with less aggressive epinephrine response to low BG, while our model demonstrated that this effect is primarily due to a less aggressive onset of counterregulation and only partly due to lower maximal counterregulation response. This result refines research findings that associate risk for SH with "impaired glucose counterregulation" (Cryer and Gerich, 1985; Gerich, 1988; White et al., 1983) clarifying one dimension of this counterregulation impairment. As a result, our model may shed light not only on which subjects may be poor candidates for intensive insulin therapy because of vulnerability to $\mathrm{SH}$; but also why they may be so (in functionally mechanistic terms).

\section{Acknowledgement}

This study is supported by the National Institutes of Health grants RO1 DK51562, RO1 DK28288, RROO847, by the National Science Foundation NSF DIR-8920162 and by the Bulgarian Research Fund Grant MM706. We thank the University of Virginia General Clinical Research Center for the support during the study.

\section{References}

Amiel, S. A., Sherwin, R. S., Simonson, D. C. and Tamborlane, W. V. (1979) Effect of intensive insulin therapy on glycemic thresholds for counterregulatory hormone release. Diabetes, 37, pp. $901-907$.

Amiel, S. A., Tamborlane, W. V., Simonson, D. C. and Sherwin, R. S. (1987) Defective glucose counter- regulation after strict glycemic control of insulin-dependent diabetes mellitus. New England Journal of Medicine, 316, pp. 1376-1383.

Bergman, R. N., Ider, Y. Z., Boeden, C. R. and Cobelli, C. (1979) Quantative estimation of insulin sensitivity. American Journal of Physiology, 236, pp. E667-E667.

Bergman, R. N.. Prager, R., Volund, A. and Olelsky, J. M. (1987) Equivalence of the insulin sensitivity index in man derived by the minimal model method and the euglycemic glucose clamp. Journal of Clinical Investigation, 79, pp. 790-800.

Bolli, G. B., DeFeo, P., DeCosmo, S., Perriello, G., Ventura, M. M., Massi Benedetti, M., Sateusario, F.. Gerich, H. E. and Brunetti, P. (1984) A reliable and reproducible test for adequate glucose counter- regulation in type I diabetes mellitus. Diabetes, $\mathbf{3 3}$, pp. $732-737$.

Brier, D. M., Arnold, K. J., Sherman, W. R., Holland, W. H., Holmes, W. F. and Kipnis, D. M. (1997) In-vivo measurement of glucose and alanine metabolism with stable isotopic tracers. Diabetes, 26, pp. 1005-1015.

Carson, E. R. and Cramp, D. G. (1976) A systems model of blood glucose control. International Journal of Biomedical Computing, 7, pp. 21-34.

Cobelli, C., Pacini, G., Toffolo, G. and Sacca, L. (1986) Estimation of insulin sensitivity and glucose clearance from minimal model: new insights from labeled IVGTT. American Joumal of Physiology, 250, pp. E591-598.

Cobelli, C., Brier, D. M. and Ferrannini, E. (1990) Modeling glucose metabolism in man: theory and practice. Hormone and Metabolic Research - Supplement, 24, pp. 1-10.

Cryer, P. E. and Gerich, J. E. (1985) Glucose counterregulation, hypoglycemia and intensive therapy of diabetes mellitus. New England Journal of Medicine, 313, pp. 232-241.

DCCT Study Group (1991) Epidemiology of severe hypoglycemia in the diabetes control and complications trial. American Journal of Medicine, 90 , pp. $450-459$.

DCCT Study Group (1993) The effect of intensive treatment of diabetes on the development and progression of long-term complications in IDDM, New England Journal of Medicine, 329, pp. 977-986.

Gerich, J. E. (1988) Glucose counterregulation and its impact on diabetes mellitus. Diabetes, 37, pp, $1608-1617$.

Guyton, J. R., Foster, R. O., Soeldner, J. S., Tan, M. H., Kahn, C. B., Koncz, L. and Gleason, R. E. (1978) A model of 
glucose-insulin homeostasis in man that incorporates the heterogeneous fast pool theory of pancreatic insulin release. Diabetes, 27, pp. $1027-1042$.

Hetenyi, G.. Ninomiya, R. and Wrenshall, G. A. (1966) Glucose production rates in dogs determined by two different tracers and tracer methods. Journal of Nuclear Medicine, 7. pp. $454-463$.

Insel, P. A., Liljenquist, J. E.. Tobin, J. D., Sherwin, R. S. Watkins, P., Andres, R. and Berman. M. (1975) Insulin control of glucose metabolism in man. A new kinetic analysis. Journal of Clinical Investigation, 55. pp. 1057-1066.

Johnson, M. L. and Frasier, S. G. (1985) Nonlinear least squares analysis. Methods of Enzymology, 117, pp. 301-342.

Kvalseth. T. O. (1985) Cautionary note about R squared. The American Statistician, 39, pp. 279-285.

Mari. A. (1997) Assessment of insulin sensitivity with minimal model: role of model assumptions. American Journal of Physiology. 272, pp. E925-E934.

Press. W. H., Flannery, B. P., Teukolsky, S. A. and Vetterling, W. T. (1989) Numerical Recipes: The Art of Scientific Computing (Fortran Version). Cambridge: Cambridge University Press, pp. 551-552.
Quon, M. J., Cochran, C., Taylor, S. I. and Eastman, R. C. (1994) Non-insulin mediated glucose disappearance in subjects with IDDM. Discordance between experimental results and minimal model analysis. Diabetes, 43, pp. 890-896.

Steele, R., Rostami, H. and Altszuler, N. (1974) A two-compartment calculator for the dog glucose pool in the nonsteady state. Federation Proceedings, 33, pp. 1869-1876.

Straume, M., Frasier-Cadoret, S. G. and Johnson, M. L. (1991) Least-squares analysis of fluorescence data. In: Lakowicz, J.R. ed. Topics in Fluorescence Spectroscopy, Vol. 2: Principles. New York: Plenum. 177-241.

White, N. H., Skor, D. A., Cryer, P. E., Bier, D. M., Levandoski, L. and Santiago, J. V. (1983) Identification of type I diabetic patients at increased risk for hypoglycemia during intensive therapy. New England Joumal of Medicine, 308, pp. $485-491$.

Yamasaki, Y., Tiran, J. and Albisser. A. M. (1984) Modelin glucose disposal in diabetic dogs fed mixed meals. American Journal of Physiology, 246, pp. E52-E61. 


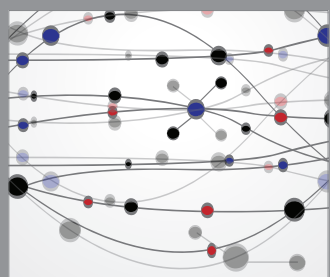

The Scientific World Journal
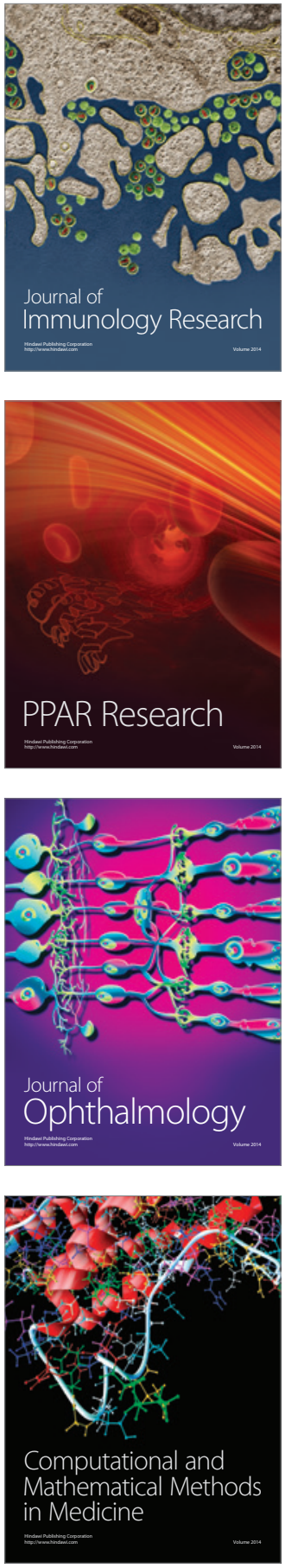

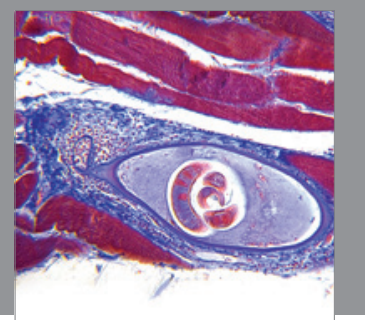

Gastroenterology

Research and Practice
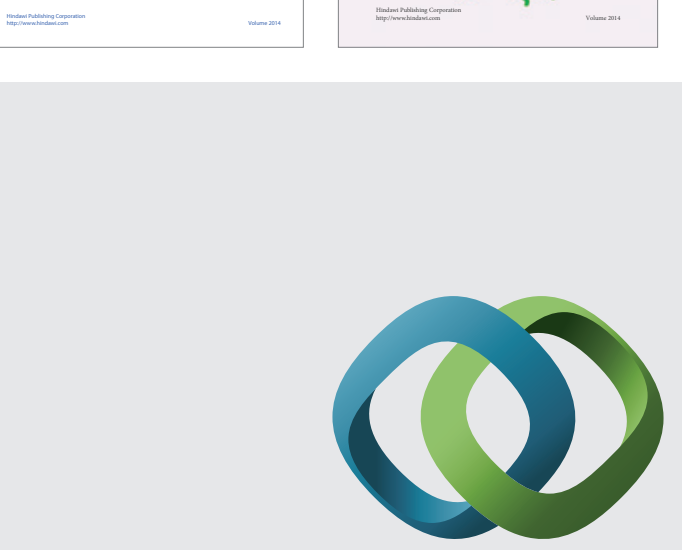

\section{Hindawi}

Submit your manuscripts at

http://www.hindawi.com
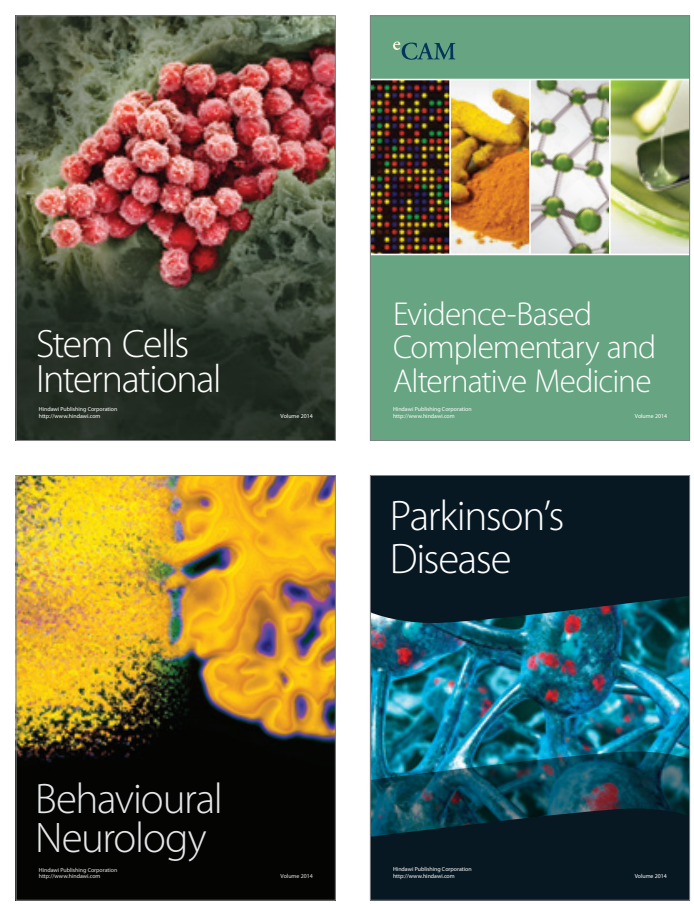

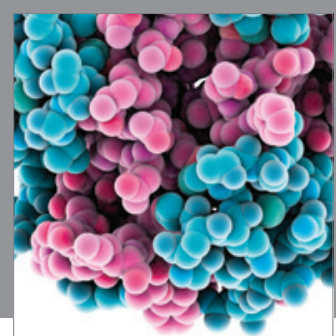

Journal of
Diabetes Research

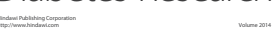

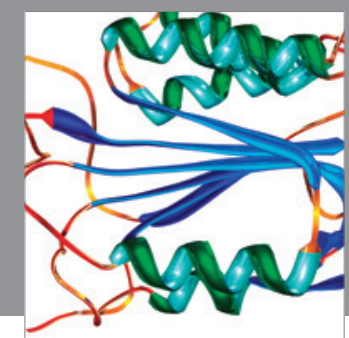

Disease Markers
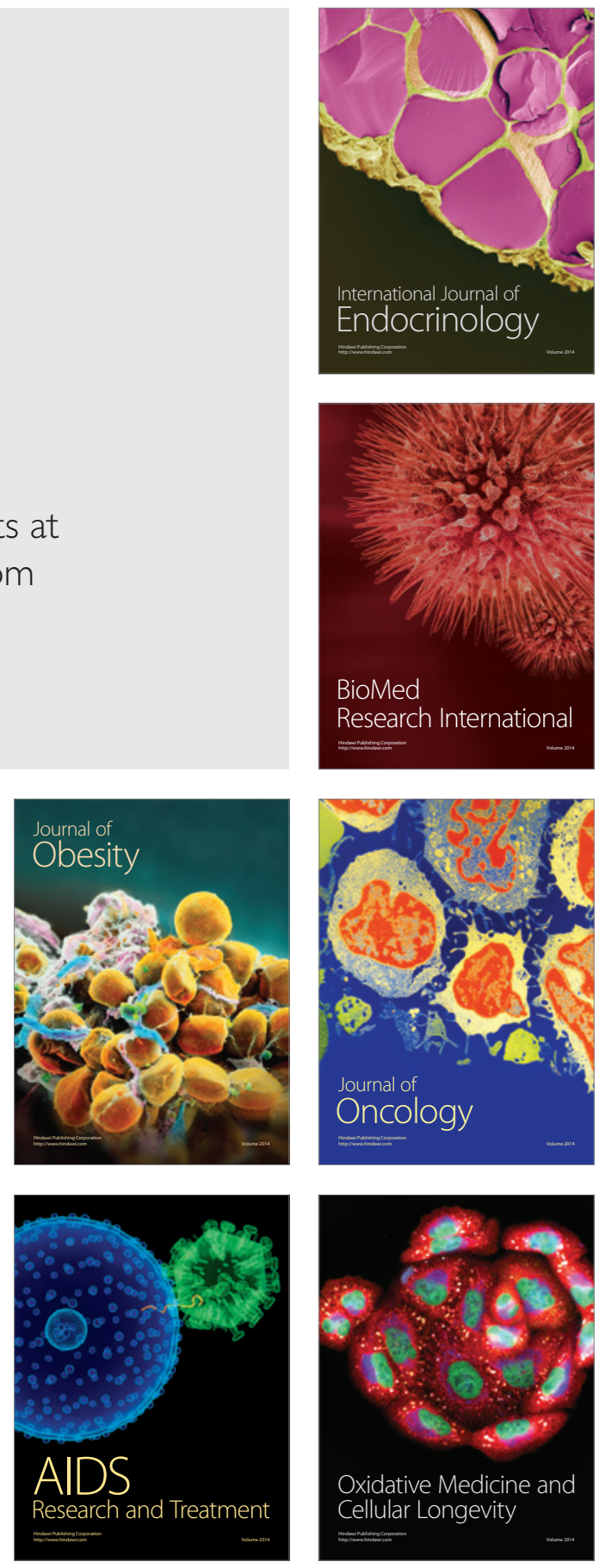